\title{
Moringa oleifera Supplemented Diets Prevented Nickel-Induced Nephrotoxicity in Wistar Rats
}

\author{
O. S. Adeyemi and T. C. Elebiyo \\ Department of Biological Sciences, Landmark University, Omu-Aran 370102, Kwara State, Nigeria \\ Correspondence should be addressed to O. S. Adeyemi; yomibowa@yahoo.com
}

Received 18 June 2014; Accepted 4 September 2014; Published 11 September 2014

Academic Editor: Joel B. Mason

Copyright ( 12014 O. S. Adeyemi and T. C. Elebiyo. This is an open access article distributed under the Creative Commons Attribution License, which permits unrestricted use, distribution, and reproduction in any medium, provided the original work is properly cited.

\begin{abstract}
Background. The Moringa oleifera plant has been implicated for several therapeutic potentials. Objective. To evaluate whether addition of $M$. oleifera to diet has protective effect against nickel-induced nephrotoxicity in rats. Methodology. Male Wistar rats were assigned into six groups of five. The rats were given oral exposure to $20 \mathrm{mg} / \mathrm{kg}$ nickel sulphate $\left(\mathrm{NiSO}_{4}\right)$ in normal saline and sustained on either normal diet or diets supplemented with Moringa oleifera at different concentrations for 21 days. 24 hours after cessation of treatments, all animals were sacrificed under slight anesthesia. The blood and kidney samples were collected for biochemical and histopathology analyses, respectively. Results. $\mathrm{NiSO}_{4}$ exposure reduced the kidney-to-body weight ratio in rats and caused significant elevation in the levels of plasma creatinine, urea, and potassium. Also, the plasma level of sodium was decreased by $\mathrm{NiSO}_{4}$ exposure. However, addition of $M$. oleifera to diets averted the nickel-induced alteration to the level of creatinine and urea. The histopathology revealed damaged renal tubules and glomerular walls caused by $\mathrm{NiSO}_{4}$ exposure. In contrast, the damages were ameliorated by the $M$. oleifera supplemented diets. Conclusion. The addition of M. oleifera to diet afforded significant protection against nickel-induced nephrotoxicity.
\end{abstract}

\section{Introduction}

The Moringa oleifera is a significant medicinal plant belonging to the family Moringaceae. The M. oleifera is recognized for its vast therapeutic properties since ancient times. It is also known as drumstick tree or horseradish tree; the leaves are very beneficial and offer important source of beta-carotene, vitamin $\mathrm{C}$, protein, iron, and potassium [1]. The M. oleifera plant is native to the Indian subcontinent and has been used by the Indians for almost 5000 years [2]. M. oleifera tree can grow well in the humid tropic or hot dry land and it can survive in harsh climatic condition including destitute soil $[2,3]$. The root, bark, gum, leaf, pods, flowers, seed, and seed oil are used in traditional medicine for treatment of various ailments [4]. The leaves, flowers, roots, gums, fruits, and seeds of $M$. oleifera are extensively used in the treatment of inflammation, cardiovascular dysfunction, liver disease, and hematological and renal malfunction [5-7]. The leaves of M. oleifera $(\mathrm{g} / \mathrm{g})$ have the calcium four times that of milk, vitamin C seven times that of oranges, and potassium three times that of bananas, three times the iron of spinach, four times the vitamin $\mathrm{A}$ in carrots, and two times the protein in milk $[8,9]$. Studies have attributed the medicinal benefits of $M$. oleifera to its anti-inflammatory, antioxidant, and antipathogenic constituents [10, 11]. Also, the strong antioxidant and scavenging ability of $M$. oleifera has been linked to chemoprevention of diseases like cancer [12].

Research efforts have continued to unearth the medicinal and nutritional potential of $M$. oleifera in several significant ways. The M. oleifera plant has been used as antispasmodic, stimulant, cough expectorant, and diuretic agent [13]. The use of M. oleifera as diuretic agent is applicable in the treatment of prostatitis, kidney stones, bladder ache, scalding urine, water retention, and obesity [14]. In a separate study, the extract of $M$. oleifera showed antinephrotoxic effect against DMBAinduced renal cancer [15]. In a similar work by Owolabi et al., [16], M. oleifera extracts reduced the severity of lead-induced nephrotoxicity. 
In furtherance of exploring and accentuating the medicinal potential of $M$. oleifera in protein against heavy metal toxicity, the present study evaluated the nephroprotective capacity of $M$. oleifera supplemented diets in Wistar rats orally administered with nickel sulphate $\left(\mathrm{NiSO}_{4}\right)$.

\section{Methods}

2.1. Chemicals and Reagents. All chemicals and reagents used were of analytical grade. Commercial reagent kits for the determination of plasma urea, creatinine, potassium, sodium, and protein were either as supplied by Randox diagnostic laboratory, Crumlin, UK, or the Agape laboratory, Switzerland.

2.2. Experimental Animals. Thirty male rats of Wistar strain of weight between 190 and $200 \mathrm{~g}$ were obtained from the Experimental Animal Farm at the Department of Biochemistry, University of Ilorin, Ilorin, Nigeria. The Wistar rats were housed in plastic animal cages in a well-ventilated experimental room. Rats were allowed to acclimatize for a period of 14 days before the commencement of treatments. During the period of acclimatization, the animals had unlimited access to rat feed pellets and clean water. Handling of animals was in accordance with relevant institutional and ethical guidelines as approved for scientific study.

2.3. Moringa oleifera. The leaves of Moringa oleifera were harvested at the Landmark University Farm, Omu-Aran, Nigeria. The leaves were identified and authenticated by Mr. Bolu Ajayi at the Herbarium Unit, Department of Plant Biology, University of Ilorin, Ilorin, Nigeria. Specimen voucher number is VIH 001/1011.

The $M$. oleifera leaves were air-dried and ground into powder. The powdered material was stored at room temperature in cool dry environment until being required for use.

2.4. Feed Formulation. The composition of the feed is as shown in Table 1.

2.5. Determination of Proximate Composition. Protein content, crude fat and fibre, moisture content, carbohydrate, and ash content were determined following established protocols as described by the Association of Official Analytical Chemists [17].

2.6. Experimental Design. Thirty male Wistar rats of weight between 195 and $200 \mathrm{~g}$ were randomly distributed into six experimental groups of five and fed as follows:

control received $\mathrm{NiSO}_{4}$ and fed normal rat chow;

group 2 received $\mathrm{NiSO}_{4}$ and fed with $5 \%$ M. oleifera supplemented diet;

group 3 received $\mathrm{NiSO}_{4}$ and fed with $10 \%$ M. oleifera supplemented diet;

group 4 received $\mathrm{NiSO}_{4}$ and fed with $15 \%$ M. oleifera supplemented diet; group 5 received normal saline and fed with $15 \% M$. oleifera supplemented diet;

group 6 received normal saline and fed with normal rat chow.

The rats were exposed to daily administration of $20 \mathrm{mg} / \mathrm{kg}$ body weight $\mathrm{NiSO}_{4}$ by oral gavage. Treatments lasted for 21 days.

2.7. Necroscopy. 24 hours after the last treatment, the animals were sacrificed under anesthesia in slight diethyl ether. Blood samples were collected by cardiac puncture into clean EDTA bottles. The blood samples were spun at $5000 \mathrm{~g}$ for 10 minutes in a refrigerated centrifuge (Anke TDL-5000B, Shanghai, China) to yield the plasma which was used for the biochemical determinations. The kidneys from each animal were excised into iced physiological solution $(\mathrm{pH}$ 7.4), weighed immediately, and fixed in buffered formalin for histopathology examinations.

2.8. Biochemical Assays. The biochemical indices were determined in rat plasma using a UV/Vis spectrophotometer (Jenway, Staffordshire, UK) where applicable. The levels of rat plasma total protein (TP), creatinine, and urea were determined using Randox assay kits (Crumlin, UK). The rat plasma electrolytes, sodium, and potassium were determined using reagent assay kits by the Agape laboratory (Switzerland). The intraday and interday coefficient of variation (CV) is less than $5 \%$.

2.9. Histopathology Examination. The rat kidney was fixed in $10 \%$ buffered neutral formalin (BNF) immediately following excision from animals. Fixed tissues were subsequently processed for histopathology examinations as previously described [18]. Capture and scoring for morphological changes were done by a pathologist blind to the treatments, the Pathology Unit, University of Ilorin Teaching Hospital, Ilorin, Nigeria.

2.10. Data analysis. Data were analysed on GraphPad Prism 3 (GraphPad Software Inc., San Diego, CA) using the one-way analysis of variance (ANOVA). Post hoc tests were conducted using the Tukey test. Data are reported as mean value \pm standard error of mean (SEM). Mean values at $P<0.05$ are significant.

\section{Results}

3.1. Proximate Composition. Diets were formulated consisting of different concentrations of $M$. oleifera. All diets used for the treatment were evaluated for proximate composition by AOAC protocols. The contents of crude fat, fibre, moisture, and ash in the normal rat chow and $M$. oleifera supplemented diets showed no significant difference (Figure 1). However, the carbohydrate content of supplemented formulated diets decreased with increasing concentration of $M$. oleifera relative to the normal diet. Conversely, protein content was higher in the M. oleifera supplemented diets. 
TABLE 1: Formulation of experimental feed.

\begin{tabular}{lcccc}
\hline Feeds component $(\mathrm{g})$ & Normal feed & 5\% Moringa oleifera & 10\% Moringa oleifera & $15 \%$ Moringa oleifera \\
\hline Sucrose & 300 & 300 & 300 & 300 \\
Cellulose & 120 & 120 & 120 & 120 \\
Soybean & 750 & 750 & 750 & 750 \\
Moringa oleifera & - & 300 & 600 & 900 \\
Vitamin mix & 150 & 150 & 150 & 12 \\
D-methionine & 12 & 12 & 918 & 12 \\
Corn starch & 1518 & 1218 & 150 & 618 \\
Soybean oil & 150 & 150 & 3000 & 150 \\
\hline Total & 3000 & 3000 & & 3000 \\
\hline
\end{tabular}

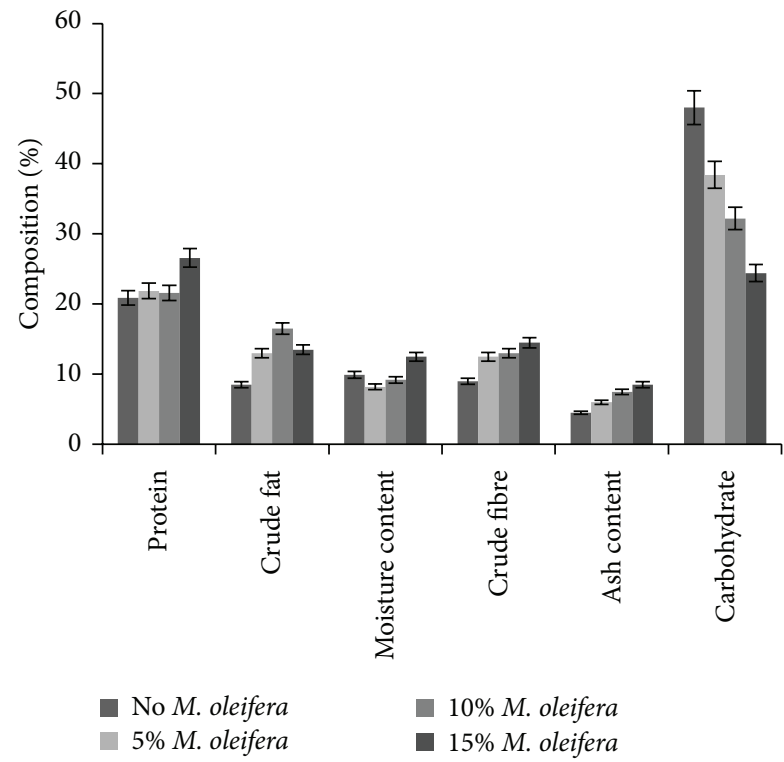

FIGURE 1: Proximate composition of experimental feeds consisting of various concentrations of Moringa oleifera leaves. Data are presented as mean values \pm standard error of mean (SEM), $n=3$.

3.2. Kidney and Body Weights. The various treatments did not evoke any appreciable change in average rat weights over the course of the exposure (Figure 2). However, there was $12 \%$ decline in rat kidney-to-body weight ratio for the group given $\mathrm{NiSO}_{4}$ and sustained on normal rat diet. Conversely, there were improvements in the kidney weight with restoration to near normal level for groups maintained on $M$. oleifera supplemented diets.

3.3. Plasma Protein. Diets containing the $15 \%$ M. oleifera caused significant increments in protein level relative to the control group (Figure 2). The other treatment groups did not show any appreciable changes.

3.4. Plasma Creatinine. In order to determine whether addition of $M$. oleifera to diets could reverse or modulate the events of nickel-induced nephrotoxicity, the level of plasma creatinine was determined. For the control group which received $\mathrm{NiSO}_{4}$ and normal rat diet, the plasma creatinine level was significantly elevated (Figure 3). On the contrary, groups given $\mathrm{NiSO}_{4}$ and sustained on $M$. oleifera supplemented diets showed significantly lower plasma creatinine level. The restoration or modulation of the rat plasma creatinine to normal level shows correlation with increasing concentration of $M$. oleifera in the diets given to rats.

3.5. Plasma Urea. Furthermore, nickel exposure in the control group sustained on normal diet led to increased $(P<$ 0.05 ) level of plasma urea (Figure 3 ). On the other hand, rats orally exposed to nickel salt and sustained on M. oleifera supplemented diets had significantly reduced level of plasma urea.

3.6. Plasma Electrolytes. Oral exposure to $\mathrm{NiSO}_{4}$ and daily sustenance on normal diet in the control group for 21 days lowered $(P<0.05)$ the level of rat plasma sodium (Figure 4$)$. However, for rats given $\mathrm{NiSO}_{4}$ and sustained on $M$. oleifera supplemented diets, there were significant elevations in levels of plasma sodium.

Also, the control group given $\mathrm{NiSO}_{4}$ and normal diet had elevated $(P<0.05)$ level of rat plasma potassium compared to groups sustained on $M$. oleifera supplemented diets in which the rat plasma potassium was appreciably maintained at near normal level.

3.7. Histopathology Examinations. The examination of rat renal sections for morphological changes revealed inimical cellular alterations in control group caused by $\mathrm{NiSO}_{4}$ exposure. The cellular alterations caused included swollen renal tubules, hyaline change, thickening of glomerular wall, mild nephritis, and necrosis (Figures 5, 6, 7, 8, 9, and 10). However, all of the cellular damages caused by $\mathrm{NiSO}_{4}$ exposure were nonexistent or ameliorated in the groups sustained on the $M$. oleifera diets.

\section{Discussion}

The $M$. oleifera has shown remarkable potential which could be harnessed for medicinal and nutritional purposes. In recent times, several studies have been able to demonstrate the medicinal significance of $M$. oleifera [19-22]. In separate 


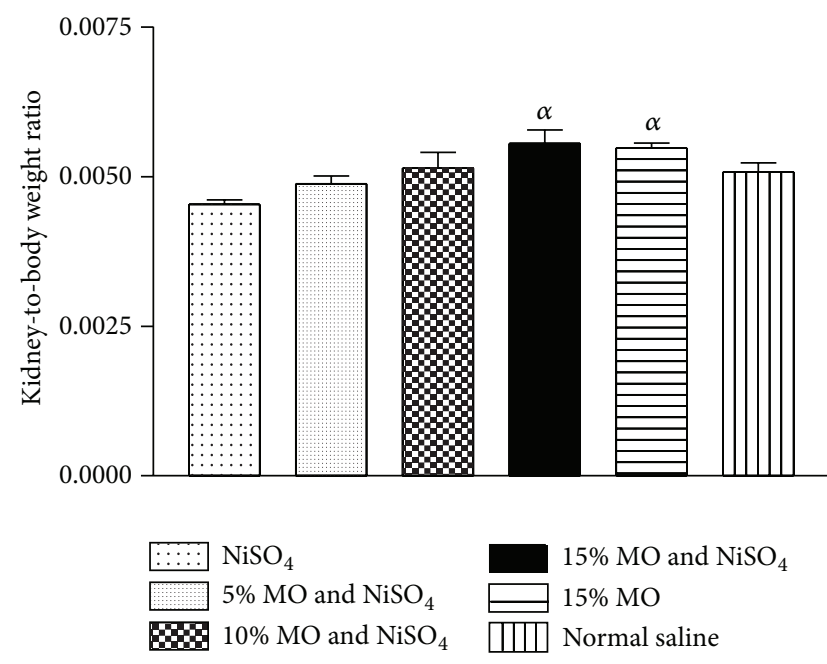

(a)

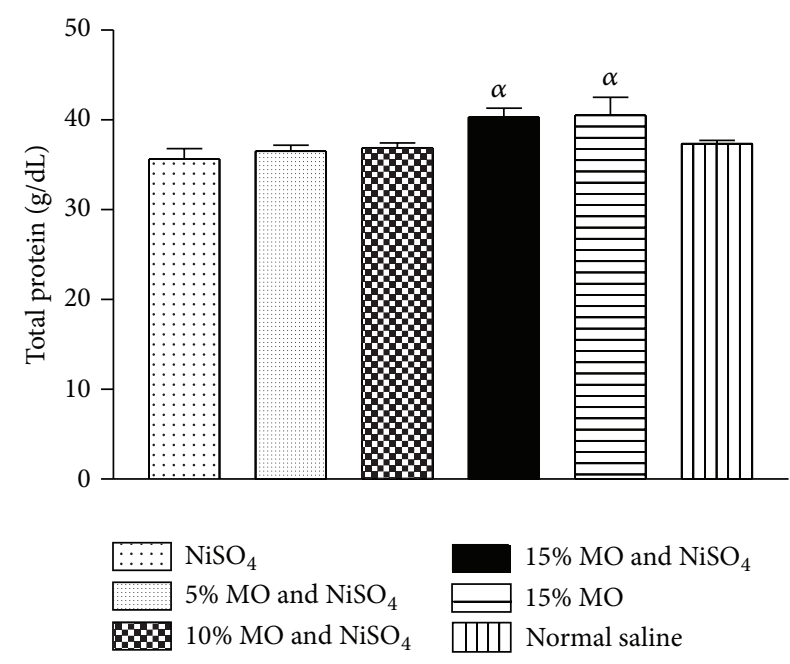

(b)

Figure 2: (a) Effect of Moringa oleifera supplemented diets on rat kidney-to-body weight ratio following exposure to nickel sulphate; (b) effect of Moringa oleifera supplemented diets on levels of rat plasma protein following exposure to nickel sulphate. Data are presented as mean values \pm standard error of mean $(\mathrm{SEM}), n=6 . \alpha$ is significantly $(P<0.05)$ relative to nickel sulphate.

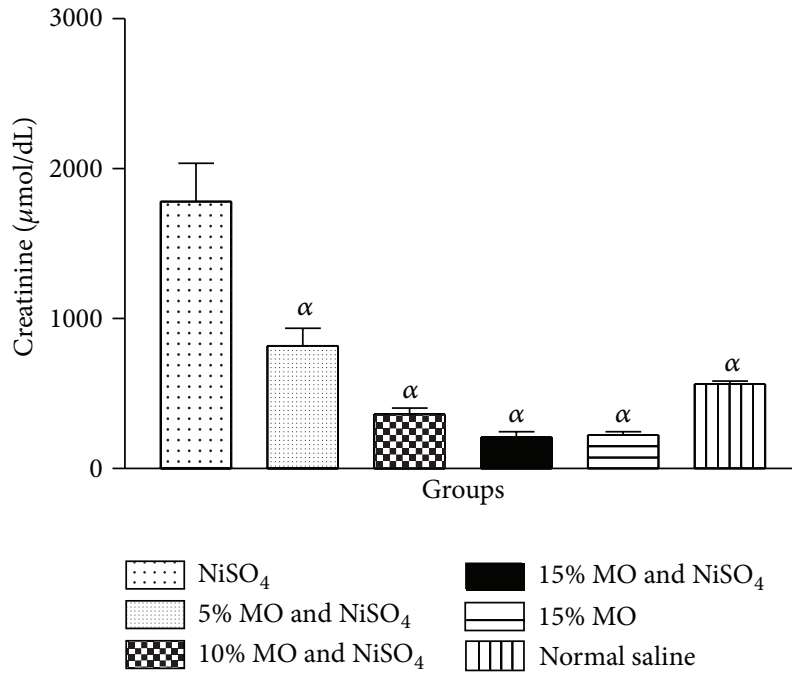

(a)

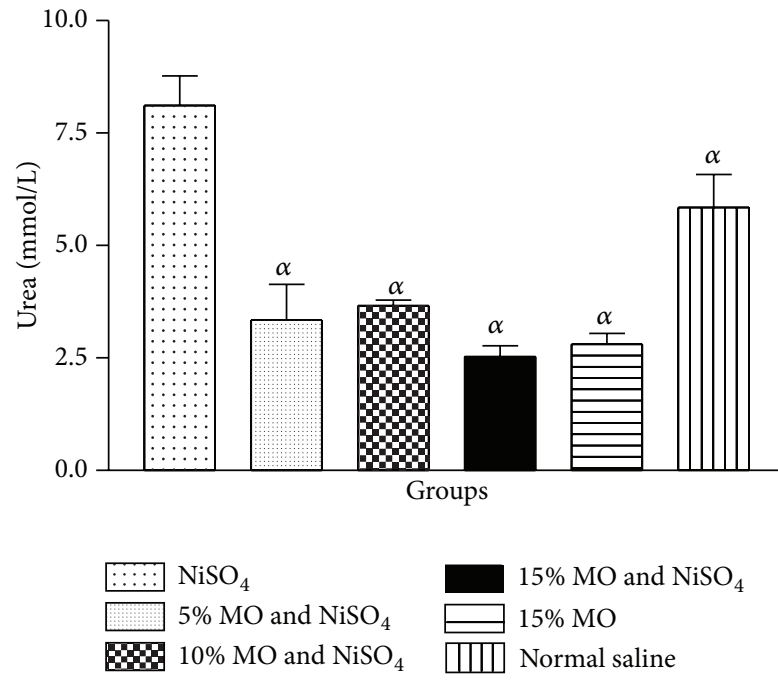

(b)

FIGURE 3: (a) Effect of Moringa oleifera supplemented diets on levels of rat plasma creatinine following exposure to nickel sulphate; (b) effect of Moringa oleifera supplemented diets on levels of rat plasma urea following exposure to nickel sulphate. Data are presented as mean values \pm standard error of mean $(\mathrm{SEM}), n=6 . \alpha$ is significantly $(P<0.05)$ relative to nickel sulphate.

studies, addition of M. oleifera to diet has revealed considerable promises as an adjunct to improving health in a variety of important ways $[22,23]$.

In furtherance of the evidence of medicinal and nutritional benefits of $M$. oleifera, diets consisting of different concentrations of $M$. oleifera were compounded and evaluated for potential of attenuating nickel-induced nephrotoxicity in rats.

Proximate analysis of the diets revealed higher protein content in the $M$. oleifera supplemented diets. The higher protein content may be due to addition of M. oleifera to the diets. Earlier studies have revealed M. oleifera as being rich in protein content $[8,9]$. To further underscore the nutritional value of $M$. oleifera diets, the proximate analysis showed reduced carbohydrate content with increasing percentage of $M$. oleifera. This may imply nutritional relevance for management of diabetics or related metabolic disorder.

Measurement of body weight and/or organ weight may be used to evaluate toxic events due to exposure to a toxicant. Alteration in body weight and/or organ to body weight ratio 


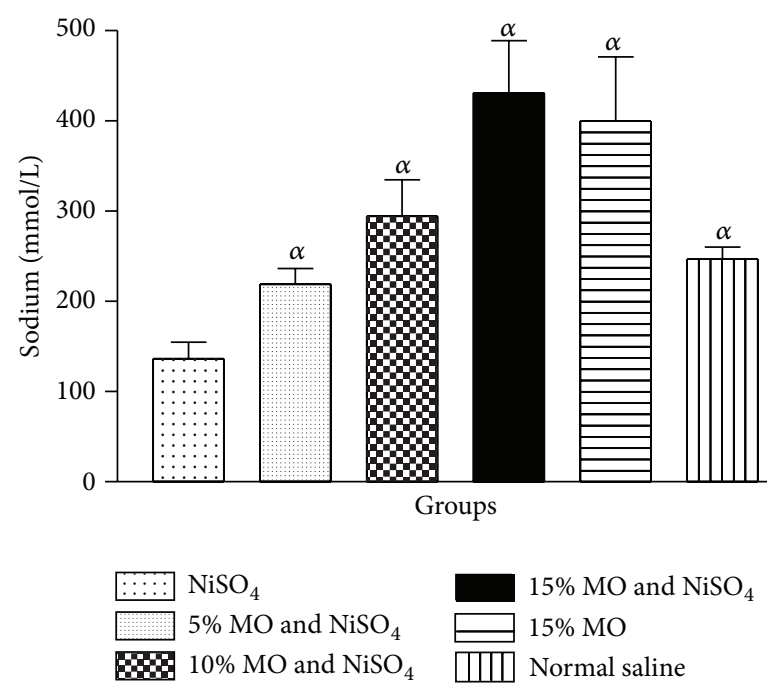

(a)

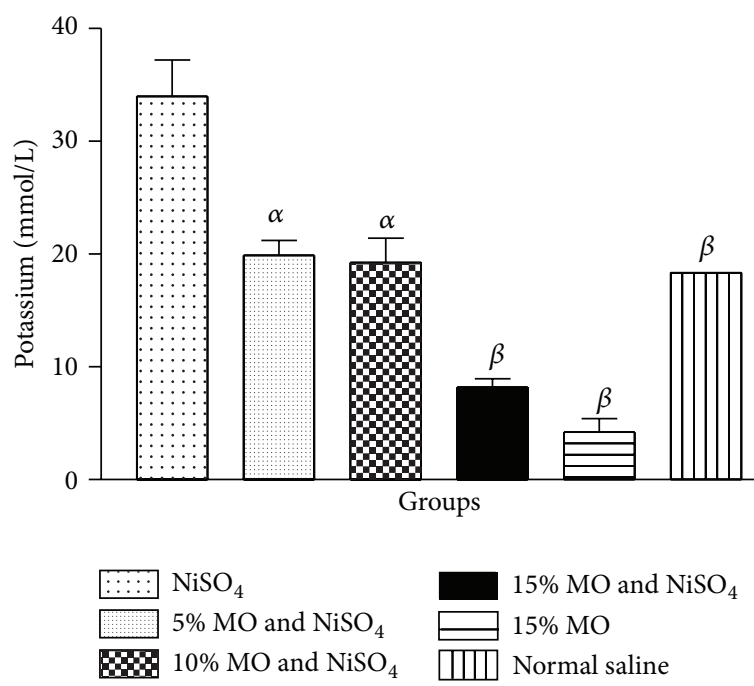

(b)

FIGURE 4: (a) Effect of Moringa oleifera supplemented diets on levels of rat plasma sodium following exposure to nickel sulphate; (b) effect of Moringa oleifera supplemented diets on levels of rat plasma potassium following exposure to nickel sulphate. Data are presented as mean values \pm standard error of mean (SEM), $n=6 . \alpha$ is significantly $(P<0.05)$ relative to nickel sulphate; $\beta$ is significantly $(P<0.01)$ relative to nickel sulphate.

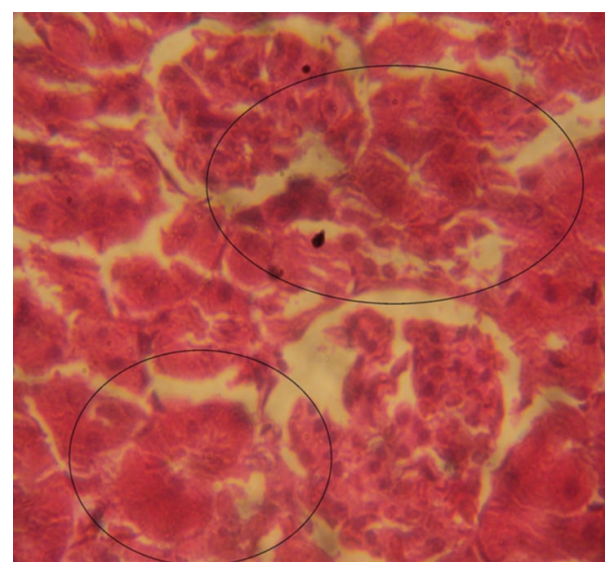

Figure 5: Photomicrographs of rat renal sections following oral exposure to nickel sulphate and sustenance on normal diets. H\&E staining $(\times 400)$.

has been shown to imply toxicity arising from exposure to a toxicant $[24,25]$. No appreciable weight change was recorded for all treatment groups. However, declining kidney-tobody weight ratio in the control group given $\mathrm{NiSO}_{4}$ and normal diet was improved significantly in groups exposed to $\mathrm{NiSO}_{4}$ and $M$. oleifera supplemented diets. This could be an indication that $M$. oleifera addition to diet was able to attenuate the adverse effect of $\mathrm{NiSO}_{4}$ administration on rat renal tissues.

On the other hand, rat plasma protein levels were not appreciably affected by $\mathrm{NiSO}_{4}$ exposure. However, sustaining animals on diets containing $15 \% \mathrm{M}$. oleifera increased the protein level. This may be due to the fact that $M$. oleifera has a high content of protein $[8,9]$. This is even more plausible if

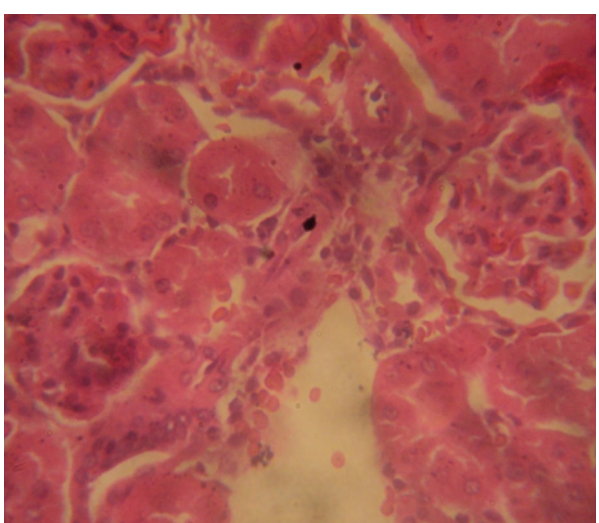

FIGURE 6: Photomicrographs of rat renal sections following oral exposure to nickel sulphate and sustenance on 5\% Moringa oleifera supplemented diets. H\&E staining $(\times 400)$.

consideration is given to the proximate composition data in which the $15 \%$ M. oleifera diet had the highest protein content.

Plasma levels of creatinine and urea are among the major biochemical indices commonly used to evaluate renal functions $[18,26]$. Creatinine is a by-product of muscle metabolism and under normal physiological condition the amount excreted per day is constant and correlates with body mass [26]. Conversely, when creatinine level is elevated due to retention in the blood, it could be used to evaluate glomerular filtration rate [18]. Urea, on the other hand, is formed as means to rid body of nitrogenous waste from protein degradation. It is formed in the liver and excreted by the kidney in urine. Elevated plasma urea level has been linked to reduced renal function $[18,26]$. 


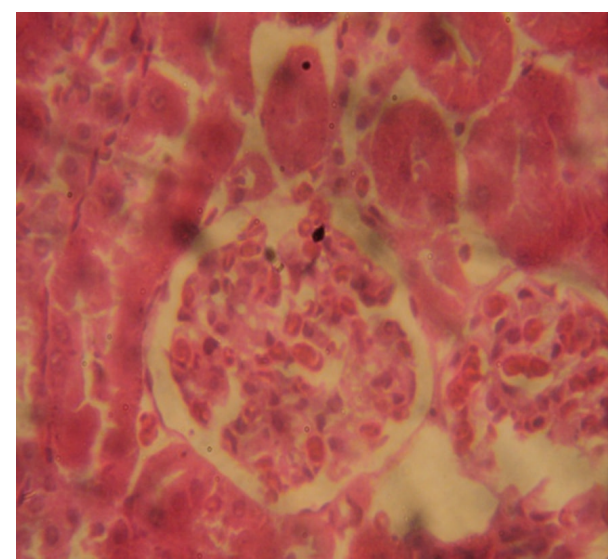

Figure 7: Photomicrographs of rat renal sections following oral exposure to nickel sulphate and sustenance on 10\% Moringa oleifera diets. H\&E staining $(\times 400)$.

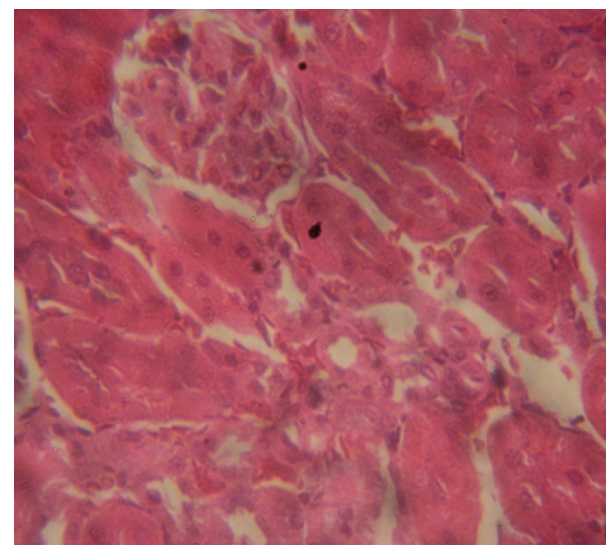

Figure 8: Photomicrographs of rat renal sections following oral exposure to nickel sulphate and sustenance on $15 \%$ Moringa oleifera diets. H\&E staining $(\times 400)$.

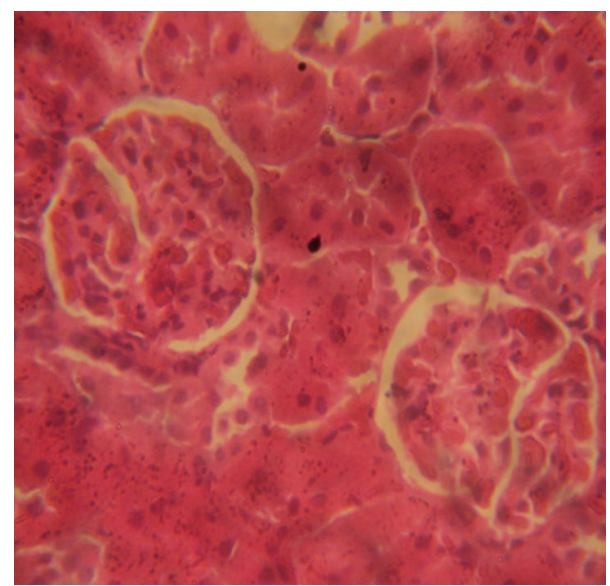

FIGURE 9: Photomicrographs of rat renal sections following oral exposure to normal saline and sustenance on 15\% Moringa oleifera diets. H\&E staining $(\times 400)$.

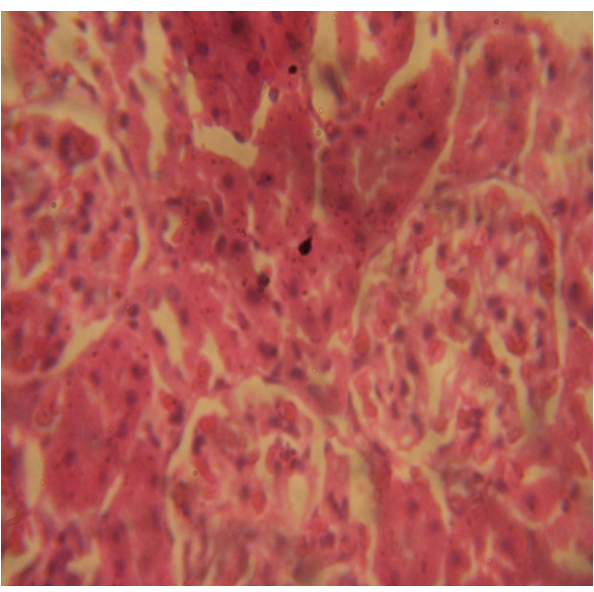

Figure 10: Photomicrographs of rat renal sections following oral exposure to normal saline and sustenance on normal diets. H\&E staining $(\times 400)$.

In the present study, levels of plasma creatinine and urea were determined. The oral exposure of $\mathrm{NiSO}_{4}$ in rats caused significant elevation to the plasma levels of creatinine and urea in a manner reminiscent of compromised renal integrity. However, the feeding of diets containing different concentrations of $M$. oleifera to rats prevented the elevation of the indices for renal dysfunction. These findings are in support of previous studies which showed that M. oleifera offered nephro- and hepatoprotection [15, 20, 21, 27]. Also, in a recent study, the protective effect of $M$. oleifera against lead-induced nephrotoxicity has been demonstrated [16]. The evidences are demonstrations which strengthen further the belief that addition of $M$. oleifera to diets could improve health status.

To further evaluate nephroprotective effect of diets containing M. oleifera, we determined the level of rat plasma electrolytes. Our results revealed significant alteration to levels of rat plasma electrolytes caused by oral exposure to $\mathrm{NiSO}_{4}$. On the contrary, the significant reductions in levels of plasma sodium and elevations in potassium level caused by $\mathrm{NiSO}_{4}$ were averted by $M$. oleifera supplemented diets. The maintenance of normal levels of plasma electrolytes is crucial to homeostatic balance. Perturbation of plasma electrolyte balance may affect the $\mathrm{pH}$, osmolality, and blood volume with adverse impact on the kidney and other related body organs. $M$. oleifera has been shown to possess diuretic effect $[13,14]$ and this may have contributed to protecting against homeostatic imbalance imposed by nickel exposure. Moreover, the maintenance of normal level of plasma sodium and potassium by $M$. oleifera diet is consistent with reported therapeutic potential including the improvement of the health of renal tissues and general well-being [20, 21]. Although, at 10 and $15 \%$ M. oleifera supplementation, there were increases or decreases in the level of sodium and potassium, respectively, the alterations were found not to be significant when compared to the untreated group given normal saline and $0 \%$ M. oleifera diet. 
The histopathology of renal sections revealed inimical cellular lesions caused by $\mathrm{NiSO}_{4}$ exposure in rats. The hyaline change, thickening of glomerular wall, mild nephritis, and necrotic areas caused by $\mathrm{NiSO}_{4}$ were conspicuously absent or attenuated in the groups fed on diets containing M. oleifera. The histopathology presentations support the biochemical findings and confirm further the medicinal protection afforded by $M$. oleifera against drug-induced tissue damage $[15,16,19,21,27]$.

To our knowledge, this is the first study which revealed that the addition of $M$. oleifera to diet protected rat kidney against the toxic events caused by $\mathrm{NiSO}_{4}$ exposure. The data are further scientific demonstration which underpins the medicinal and nutritional potential of $M$. oleifera. For people working in the mining industries or other related sectors, where the frequency of exposure to heavy metals is high, consumption of diet containing $M$. oleifera may help protect against occupational health risks.

\section{Highlights}

(1) Male Wistar rats were daily exposed to oral administration of $\mathrm{NiSO}_{4}$ and sustained on either normal diets or Moringa oleifera supplemented diets.

(2) In the control group which was sustained on normal diets, $\mathrm{NiSO}_{4}$ exposure altered the levels of plasma metabolites including urea, creatinine, and electrolytes in a manner that is reminiscent of impaired renal function.

(3) In the other treatment groups sustained on Moringa oleifera supplemented diets, there were no alterations to the levels of plasma urea, creatinine, and electrolytes revealing nephroprotective potential of the supplemented diets.

(4) Oral exposure of $\mathrm{NiSO}_{4}$ caused damage to renal tubules and glomerular walls. However, Moringa oleifera supplemented diets protected against the renal damage.

(5) The addition of Moringa oleifera to diet protected against nickel-induced nephrotoxicity.

\section{Conflict of Interests}

The authors declare that there is no conflict of interests regarding the publication of this paper.

\section{Acknowledgment}

The authors thank the laboratory staff of the Department of Biological Sciences, Landmark University, Nigeria, for the technical support.

\section{References}

[1] A. R. Verma, M. Vijayakumar, C. S. Mathela, and C. V. Rao, "In vitro and in vivo antioxidant properties of different fractions of
Moringa oleifera leaves," Food and Chemical Toxicology, vol. 47, no. 9, pp. 2196-2201, 2009.

[2] J. F. Morton, "The horseradish tree, Moringa pterygosperma (Moringaceae) - a boon to arid lands?" Economic Botany, vol. 45, no. 3, pp. 318-333, 1991.

[3] F. Anwar, M. Ashraf, and M. I. Bhanger, "Interprovenance variation in the composition of Moringa oleifera oilseeds from Pakistan," Journal of the American Oil Chemists' Society, vol. 82, no. 1, pp. 45-51, 2005.

[4] A. Odebiyi and E. A. Sofowora, "Pytochemical screenings of Nigerian medicinal plants part 11," Lyodia, vol. 44, pp. 234-246, 1999.

[5] U. K. Mazumder, M. Gupta, S. Chakrabarti, and D. Pal, "Evaluation of hematological and hepatorenal functions of methanolic extract of Moringa oleifera Lam. root treated mice," Indian Journal of Experimental Biology, vol. 37, no. 6, pp. 612-614, 1999.

[6] K. S. Rao and S. H. Mishra, "Anti-inflammatory and antihepatotoxic activities of the rats of moringa pterygosperma geaertn," Indian Journal of Pharmaceutical Sciences, vol. 60, no. 1, pp. 1216, 1998.

[7] S. G. Mahajan and A. A. Mehta, "Effect of Moringa oleifera Lam. seed extract on ovalbumin-induced airway inflammation in guinea pigs," Inhalation Toxicology, vol. 20, no. 10, pp. 897909, 2008.

[8] W. J. Asante, I. L. Nasare, D. Tom-Dery, K. Ochire-Boadu, and K. B. Kentil, "Nutrient composition of Moringa oleifera leaves from two agro ecological zones in Ghana," African Journal of Plant Science, vol. 8, no. 1, pp. 65-71, 2014.

[9] E. I. Bamishaiye, F. F. Olayemi, E. F. Awagu, and O. M. Bamshaiye, "Proximate and phytochemical composition of Moringa oleifera leaves at three stages of maturation," Advance Journal of Food Science and Technology, vol. 3, no. 4, pp. 233-237, 2011.

[10] N. A. Kumar and L. Pari, "Antioxidant action of Moringa oleifera Lam. (drumstick) against antitubercular drugs induced lipid peroxidation in rats," Journal of Medicinal Food, vol. 6, no. 3, pp. 255-259, 2003.

[11] R. Bharali, J. Tabassum, and M. R. H. Azad, "Chemomodulatory effect of Moringa oleifera, Lam, on hepatic carcinogen metabolising enzymes, antioxidant parameters and skin papillomagenesis in mice," Asian Pacific Journal of Cancer Prevention, vol. 4, no. 2, pp. 131-139, 2003.

[12] S. E. Atawodi, J. C. Atawodi, G. A. Idakwo et al., "Evaluation of the polyphenol content and antioxidant properties of methanol extracts of the leaves, stem, and root barks of Moringa oleifera Lam," Journal of Medicinal Food, vol. 13, no. 3, pp. 710-716, 2010.

[13] M. Mbikay, "Therapeutic potential of Moringa oleifera leaves in chronic hyperglycemia and dyslipidemia: a review," Frontiers in Pharmacology, vol. 3, article 24, 2012.

[14] P. S. Kumar, D. Mishra, G. Ghosh, and G. S. Panda, "Medicinal uses and pharmacological properties of Moringa oleifera," International Journal of Phytomedicine, vol. 2, no. 3, pp. 210-216, 2010.

[15] V. Sharma and R. Paliwal, "Chemo protective role of Moringa oleifera and its isolated saponin against DMBA induced tissue damage in male mice: a histopathological analysis," International Journal of Drug Development \& Research, vol. 4, no. 4, pp. 215-228, 2012.

[16] J. O. Owolabi, O. K. Ghazal, F. E. Williams, and O. O. Gurusa, "Assessment of the prophylactic and rejuvenative effects of moringa oleifera phytochemicals extracts on lead-induced renal 
tissue disruption in adults male wistar rats models," in Proceedings of the Moringa at the Leading Edge: International Conference on Moringa Oleifera, vol. 1, pp. 1-12, 2012.

[17] AOAC, Official Methods of Analysis, Association of Official Analytical Chemists, Washington, DC, USA, 15th edition, 1995.

[18] O. S. Adeyemi and M. A. Akanji, "Psidium guajava leaf extract: effects on rat serum homeostasis and tissue morphology," Comparative Clinical Pathology, vol. 21, no. 4, pp. 401-407, 2012.

[19] L. Pari and N. A. Kumar, "Hepatoprotective activity of Moringa oleifera on antitubercular drug-induced liver damage in rats," Journal of Medicinal Food, vol. 5, no. 3, pp. 171-177, 2002.

[20] F. Anwar, S. Latif, M. Ashraf, and A. H. Gilani, "Moringa oleifera: a food plant with multiple medicinal uses," Phytotherapy Research, vol. 21, no. 1, pp. 17-25, 2007.

[21] M. Ndong, M. Uehara, S.-I. Katsumata, and K. Suzuki, "Effects of oral administration of Moringa oleifera Lam on glucose tolerance in Goto-Kakizaki and wistar rats," Journal of Clinical Biochemistry and Nutrition, vol. 40, no. 3, pp. 229-233, 2007.

[22] C. Promkum, P. Kupradinun, S. Tuntipopipat, and C. Butryee, "Nutritive evaluation and effect of Moringa oleifera pod on clastogenic potential in the mouse," Asian Pacific Journal of Cancer Prevention, vol. 11, no. 3, pp. 627-632, 2010.

[23] B. C. Johnson, Clinical Perspectives on the Health Effects of Moringa oleifera : A Promising Adjunct for Balanced Nutrition and Better Health, KOS Health Publications, 2005.

[24] O. E. Orisakwe, D. C. Husaini, and O. J. Afonne, "Testicular effects of sub-chronic administration of Hibiscus sabdariffa calyx aqueous extract in rats," Reproductive Toxicology, vol. 18, no. 2, pp. 295-298, 2004.

[25] O. S. Adeyemi, M. Fambegbe, O. R. Daniyan, and I. Nwajei, "Yoyo Bitters, a polyherbal formulation influenced some biochemical parameters in Wistar rats," Journal of Basic and Clinical Physiology and Pharmacology, vol. 23, no. 4, pp. 135138, 2012.

[26] J. L. Gross, M. J. de Azevedo, S. P. Silveiro, L. H. Canani, M. L. Caramori, and T. Zelmanovitz, "Diabetic nephropathy: diagnosis, prevention, and treatment," Diabetes Care, vol. 28, no. 1, pp. 164-176, 2005.

[27] O. Awodele, I. A. Oreagba, S. Odoma, J. A. da Silva, and V. O. Osunkalu, "Toxicological evaluation of the aqueous leaf extract of Moringa oleifera Lam. (Moringaceae)," Journal of Ethnopharmacology, vol. 139, no. 2, pp. 330-336, 2012. 


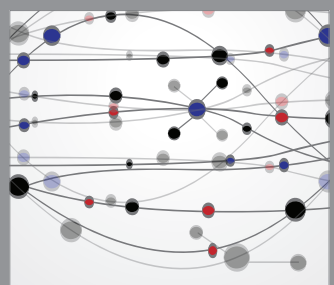

The Scientific World Journal
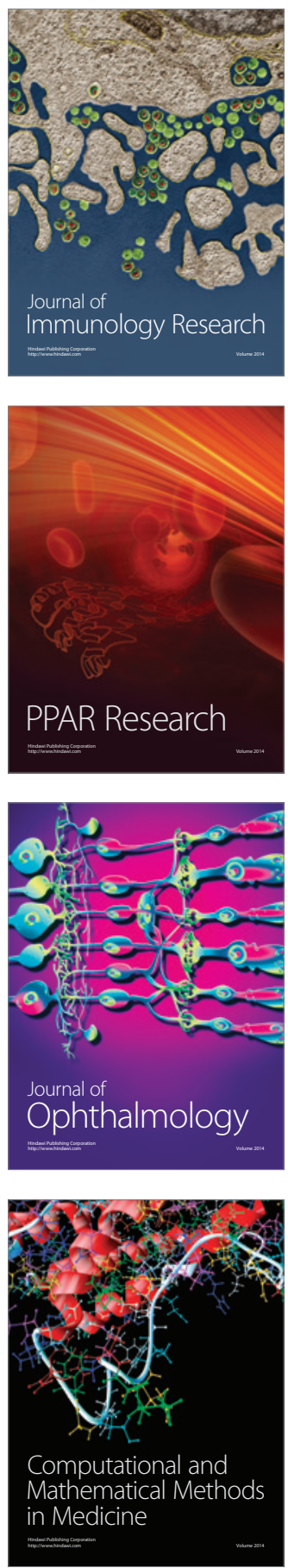

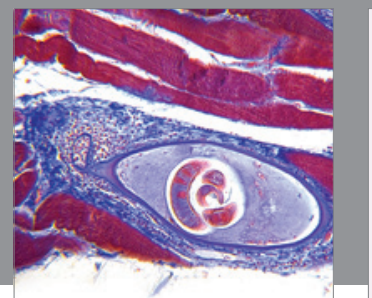

Gastroenterology

Research and Practice
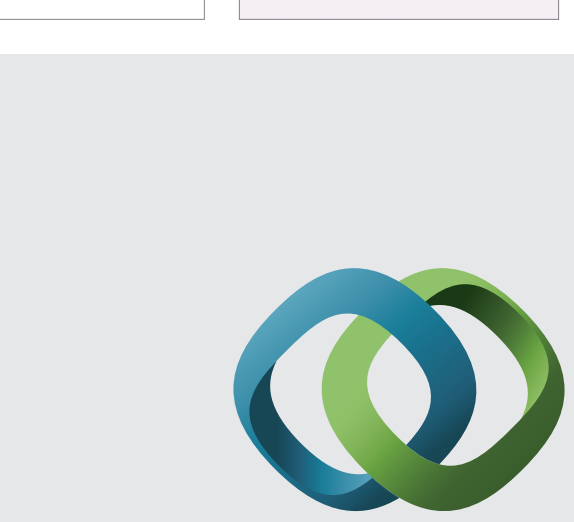

\section{Hindawi}

Submit your manuscripts at

http://www.hindawi.com
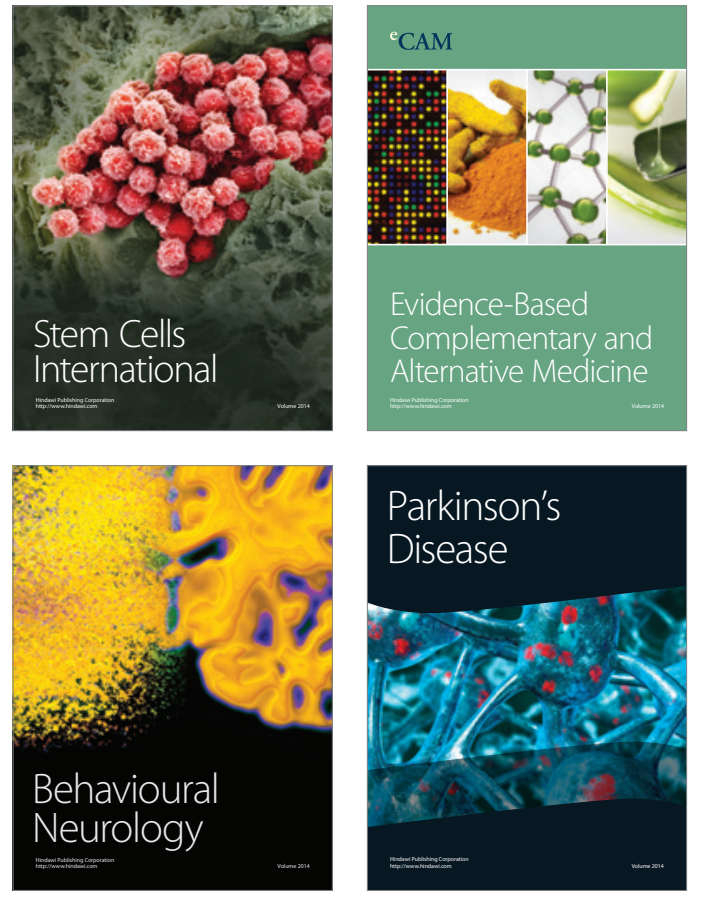
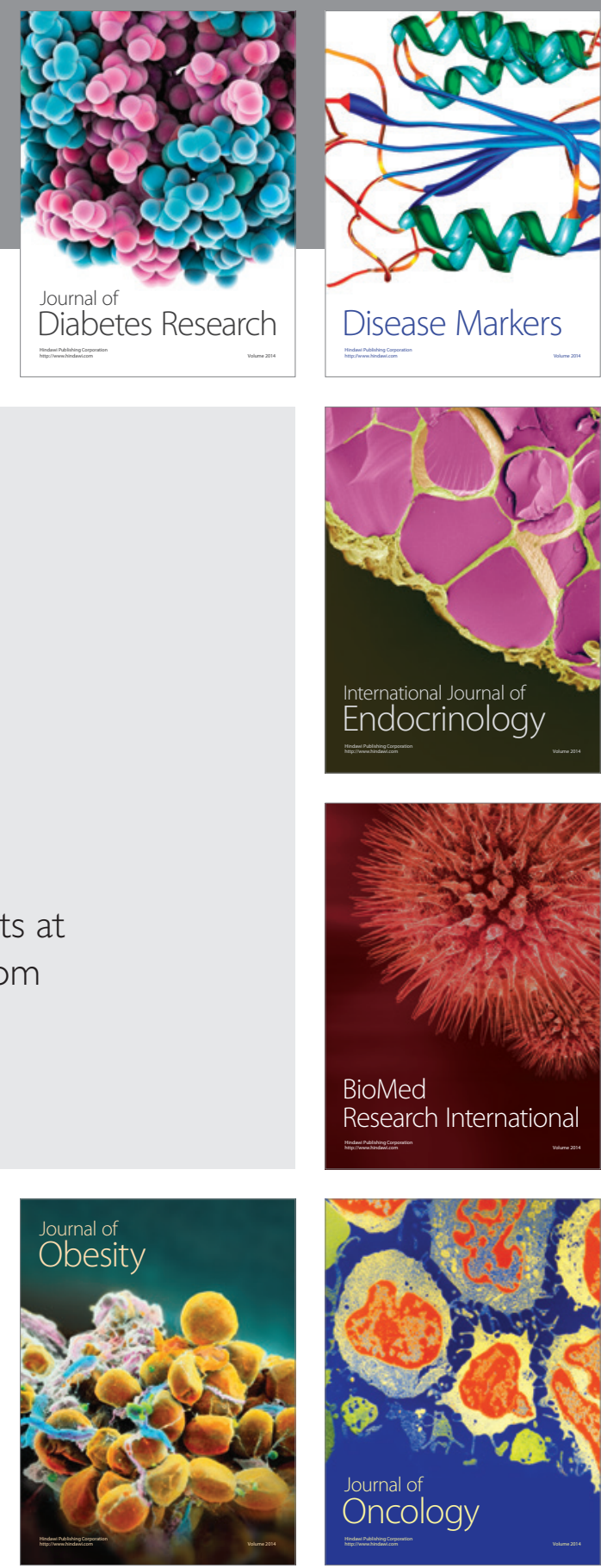

Disease Markers
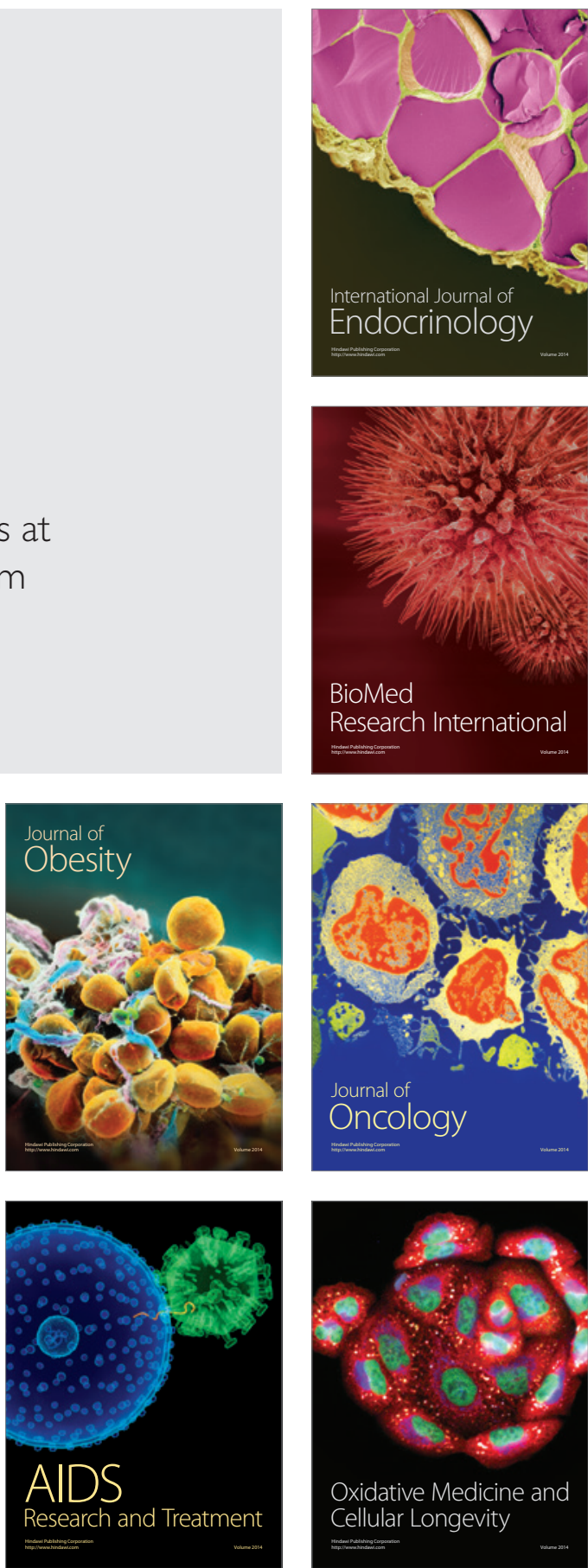